\title{
Assessment and Predicting Factors of Repeated Brain Computed Tomography in Traumatic Brain Injury Patients for Risk-Stratified Care Management: A 5-Year Retrospective Study
}

\author{
Preeda Sumritpradit, ${ }^{1}$ Thitipong Setthalikhit, ${ }^{1}$ and Sorayouth Chumnanvej ${ }^{2}$ \\ ${ }^{1}$ Acute Care Unit, Department of Surgery, Faculty of Medicine Ramathibodi Hospital, Mahidol University, Bangkok, Thailand \\ ${ }^{2}$ Neurosurgery Division, Department of Surgery, Faculty of Medicine Ramathibodi Hospital, Mahidol University, Bangkok, Thailand \\ Correspondence should be addressed to Sorayouth Chumnanvej; sorayouth.chu@mahidol.ac.th
}

Received 21 April 2016; Revised 7 August 2016; Accepted 21 August 2016

Academic Editor: Changiz Geula

Copyright (C) 2016 Preeda Sumritpradit et al. This is an open access article distributed under the Creative Commons Attribution License, which permits unrestricted use, distribution, and reproduction in any medium, provided the original work is properly cited.

Background and Objective. To determine the value of repeated brain CT in TBI cases for risk-stratified care management (RSCM) and to identify predicting factors which will change the neurosurgical management after repeated brain CTs. Methods. A 5-year retrospective study from January 2009 to August 2013 was conducted. The primary outcome was the value of repeated brain CT in TBI cases. The secondary outcome is to identify predicting factors which will change the neurosurgical management after repeated brain CTs. Results. There were 145 consecutive patients with TBI and repeated brain CT after initial abnormal brain CT. Forty-two percent of all cases $(N=61)$ revealed the progression of intracranial hemorrhage after repeated brain CT. In all 145 consecutive patients, $67.6 \%$ of cases $(N=98)$ were categorized as mild TBI. For mild head injury, $8.2 \%$ of cases $(N=8)$ had undergone neurosurgical management after repeated brain CT. Only 1 from 74 mild TBI patients with repeated brain CT had neurosurgical intervention. Clopidogrel and midline shift more than $2 \mathrm{~mm}$ on initial brain CT were significant predicting factors to indicate the neurosurgical management in mild TBI cases. Conclusion. Routine repeated brain CT for RSCM had no clinical benefit in mild TBI cases.

\section{Introduction}

Traumatic brain injury (TBI) patients with equivocal findings of brain computed tomography (CT) at the initial presentation frequently have repeated brain CTs. Repeated brain $\mathrm{CT}$ is commonly practiced at several trauma centers without protocol in place. Utilization of brain CT has increased over time; however, effects on outcome and associated risks are unknown. Brain CT may provide earlier identification for a type of traumatic brain injury. As a result, this patient will be receiving more aggressive neurosurgical intervention. In Ramathibodi Hospital, there is no protocol in place regarding repeated brain CT for TBI patients and it is controversy. So, based on physician preference and patient safety, repeated brain $\mathrm{CT}$ is still exercised. Regarding the patient safety, the risk-stratified care management (RSCM) is the interested procedure to assign a health risk status to a patient and to directly improve care management. Repeated brain CT is the option and the only one of the investigation of choice for the equivocal condition to establish TBI patient risk status as an objective tool. The apparent benefit of repeated brain CT was determined.

\section{Material and Methods}

A retrospective study of consecutive adult patients admitted to Acute Care Unit, Surgery Department, Faculty of Medicine Ramathibodi Hospital, after head injury was carried out after IRB approval. Data were collected from January 2009 to August 2013. Inclusion criteria were age more than fifteen years and duration of admission less than seventy-two hours. Patients who were treated with supportive treatment after initial brain CT were recruited. There were 145 cases potentially eligible. Variables collections including age, sex, underlying 
medical problems and medication, initial brain CT results, indication for repeated brain CT, Glasgow Coma Scale, Injury Severity Score, and treatment following repeated brain CT were determined.

2.1. Definition. Repeated brain CT was ordered by the neurosurgeons after personal assessment of TBI patient even though there were equivocal findings from initial brain CT and still no neurological deterioration. This is classified as repeated brain CT. Neurologic deterioration means alteration of neurologic status from neurological examination such as alteration of consciousness, limb weakness, lateralizing signs, and sudden appearance of severe symptoms such as headache, vomiting, and dizziness. Progressive hemorrhagic injury was identified when comparing repeated brain CT with initial brain CT. If there were any findings, even one or more of these findings will be included such as increasing in volume or size of hematoma/contusion, increasing of edema effect, or appearance of new lesions. The criteria for classification of TBI patients included mild TBI Glasgow Coma Scale $($ GCS $)=15-13$, moderate TBI GCS $=9-12$, and severe TBI GCS $\leq 8$. Regarding the surgical intervention criteria for mild TBI cases, increasing of hematoma size more than $30 \%$, increasing of hematoma volume $\geq 30 \mathrm{cc}$, surfacing location of hematoma from brain CT, asymmetrical basal cistern, and interval increasing of midline shift more than $2 \mathrm{~mm}$ are the indication for surgery after repeated CT head in mild TBI cases. All the measurement was determined by the software in PAC system. The sequential CT data sets were measured using the software, Volume Viewer Package on an Advantage Workstation 4.4 (GE Healthcare, Little Chalfont, UK) by neurosurgeons.

2.2. Statistical Analysis. Statistical analysis was done using StataCorp 2013, Stata: Statistical Software, College Station, TX: StataCorp LP, version 12 . The $P$ value $<0.05$ is the level of statistical significance.

\section{Results}

There were 145 patients with traumatic brain injury and repeated brain CT potentially eligible. Around $67.6 \%$ of all cases $(N=98)$ were categorized as mild head injury, $13.1 \%$ of all cases $(N=19)$ as moderate head injury, and $19.3 \%$ of all cases $(N=28)$ as severe head injury (Figure 1$)$. The mean (SD) age of the population was 52.0 (22.7) years (median age, 51 years; range, 15-93 years), with 71 of the 145 patients (48.9\%) being younger than 50 years of age. About $69.7 \%$ of all cases $(N=101)$ were men, with a mean (SD) ISS of 20.6 (9.2) (median ISS, 20; range, 1-75), and $44.8 \%$ of all cases $(N=65)$ were in traffic accident from Table 1 . In all 145 consecutive patients, $7.6 \%$ of cases $(N=$ 11) had neurosurgical intervention. For mild head injury, $8.2 \%$ of patients $(N=8)$ underwent immediate surgery after repeated brain CT. 74 of 98 patients had been ordered for repeated brain CT investigation. Only 1 from 74 patients (1.35\%) had neurosurgical intervention. And 24 of 98 patients had been ordered for emergency brain CT because of neurological deterioration. Seven of them (29.1\%) had undergone
TABLE 1: Demographic and clinical characteristics.

\begin{tabular}{|c|c|}
\hline Characteristic & Total $(n=145)$ \\
\hline Male & $101(69.7 \%)$ \\
\hline Age (year) & $52.0 \pm 22$ \\
\hline$>50$ years old & $71(49 \%)$ \\
\hline \multicolumn{2}{|l|}{ Mechanism } \\
\hline Motor vehicle accident & $10(6.9 \%)$ \\
\hline Motorcycle accident & $44(30.3 \%)$ \\
\hline High fall & $13(9.0 \%)$ \\
\hline Low impact fall & $55(37.9 \%)$ \\
\hline Body assault & $11(7.6 \%)$ \\
\hline Others & $12(8.2 \%)$ \\
\hline \multicolumn{2}{|l|}{ Underlying disease } \\
\hline Diabetes mellitus & $23(15.9 \%)$ \\
\hline Hypertension & $47(32.4 \%)$ \\
\hline Ischemic heart disease & $14(9.7 \%)$ \\
\hline Cerebrovascular disease & $11(7.6 \%)$ \\
\hline \multicolumn{2}{|l|}{ Medication } \\
\hline Aspirin & $26(17.9 \%)$ \\
\hline Warfarin & $6(4.1 \%)$ \\
\hline Clopidogrel & $8(5.5 \%)$ \\
\hline ISS & $20.6 \pm 9$ \\
\hline ISS > 19 & $73(50.3 \%)$ \\
\hline Brain AIS & $3.9 \pm 1$ \\
\hline AIS $>4$ & $33(22.8 \%)$ \\
\hline \multicolumn{2}{|l|}{ Result of initial brain CT } \\
\hline Epidural hematoma & $28(19.3 \%)$ \\
\hline Subdural hematoma & $92(63.4 \%)$ \\
\hline Subarachnoid hemorrhage & $81(55.9 \%)$ \\
\hline Hemorrhagic contusion & $82(56.6 \%)$ \\
\hline Intraventricular hemorrhage & $10(6.9 \%)$ \\
\hline Diffuse axonal injury & $14(9.7 \%)$ \\
\hline Skull fracture & $61(42.1 \%)$ \\
\hline Base of skull fracture & $14(9.7 \%)$ \\
\hline
\end{tabular}

operation. Comparing with conservative treatment patient group, old age, more underlying disease, and higher ISS and AIS were the conditions indicated for neurosurgical intervention. Clopidogrel (OR, 10.2 [95\% CI, 1.87-55.38]) and midline shift more than $2 \mathrm{~mm}$ on initial brain CT (OR, 11.9 [95\% CI, 2.50-57.20]) were statistical significance predicting factors for patients with mild head injury to undergo operation besides neurological deterioration in Tables 2-3. Indication for emergency CT head is based on alteration of consciousness, progressive weakness, and lateralizing signs and became confusing. Two of these patients denied surgery when neurosurgeon suggested with surgical indication and finally expired. Three patients underwent a craniotomy with clot removal; 2 patients underwent craniectomy. The remaining 1 patient underwent a burr-hole operation in Table 4. Only one patient after repeated brain CT had neurosurgical intervention who was 44-year-old female with motorcycle accident. Glasgow Coma Scale (GCS) at emergency unit was 13 and initial brain CT showed acute epidural hematoma 


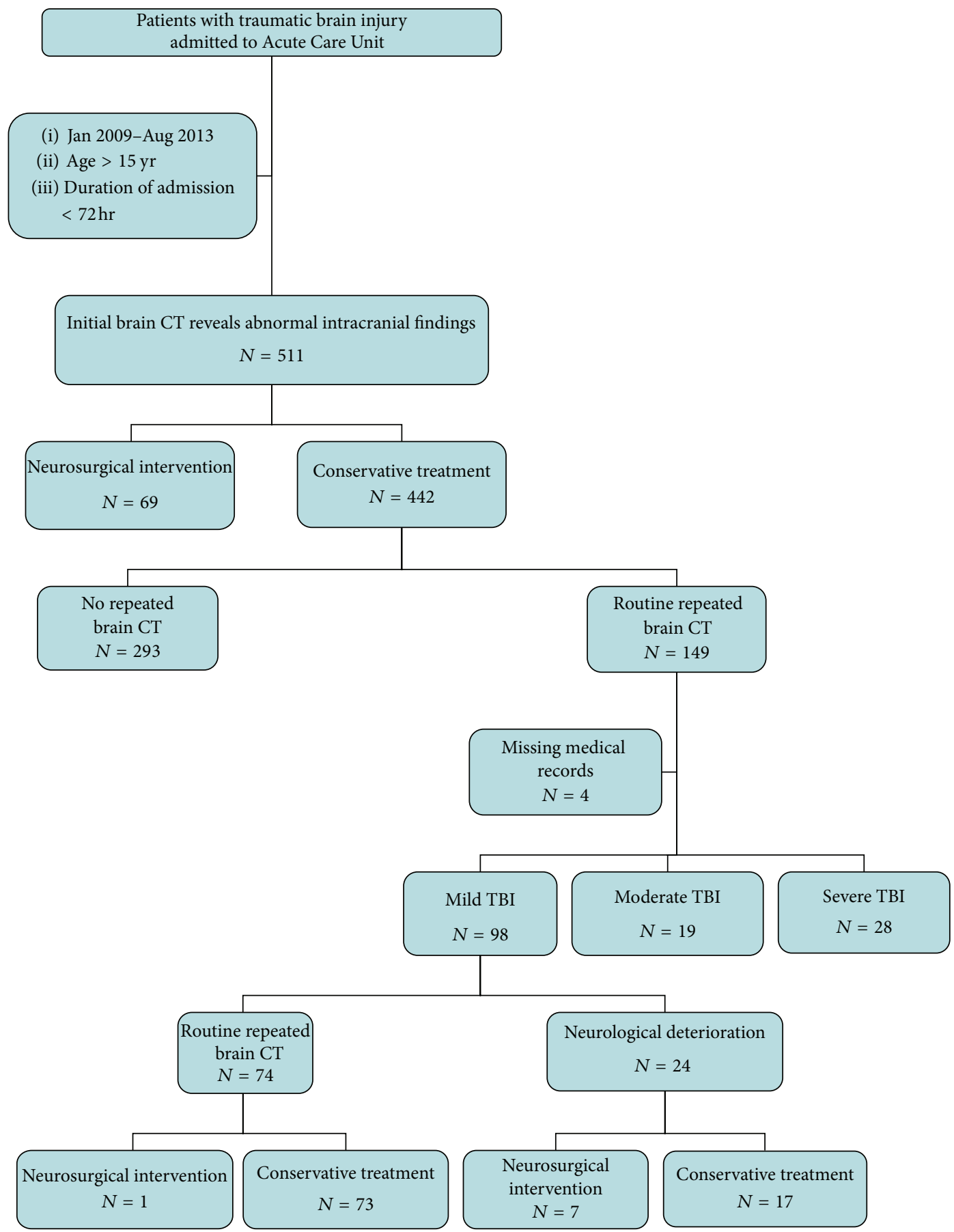

FIgURE 1: Study flow diagram.

$1.7 \mathrm{~mm}$. After conservative treatment, GCS was still the same but after repeated brain CT there was a progression of hematoma size from 1.7 to $19 \mathrm{~mm}$. Finally, this case was indicated for neurosurgical intervention. Sixty-one cases from 145 patients (42\% of cases) revealed progression of intracranial hemorrhage, and 9 patients (15\% of cases) needed neurosurgical intervention. Comparing repeated and initial brain CT, the mild TBI case was presented in Table 5 and the moderate or severe TBI was shown in Table 6. No statistical significance exists between severity of head injury and progression of hemorrhage $(P$ value $=0.186)$. Of overall 98 cases of mild TBI, about $91.8 \%(N=90)$ of cases were under conservative treatment. As a result, $8.2 \%(N=8)$ of cases underwent neurosurgical operation. 
TABLE 2: Clinical characteristics between conservative treatment group and neurosurgical treatment group after routine repeated brain CT in mild TBI (GCS 13-15).

\begin{tabular}{|c|c|c|c|}
\hline Characteristic & Conservative treatment $(n=90)$ & Neurosurgical treatment $(n=8)$ & $P$ value \\
\hline Age, mean (SD), years & $56.5(23.0)$ & $65.3(17.7)$ & 0.295 \\
\hline Age $>50 \mathrm{y}, \mathrm{N}(\%)$ & $54(60.0)$ & $6(75.0)$ & 0.478 \\
\hline Male sex, $N(\%)$ & $55(61.1)$ & $6(75.0)$ & 0.706 \\
\hline Traffic injury, $N(\%)$ & $33(36.6)$ & $1(12.5)$ & 0.256 \\
\hline \multicolumn{4}{|l|}{ Underlying disease, $N(\%)$} \\
\hline Diabetes mellitus & $17(18.8)$ & $3(37.5)$ & 0.354 \\
\hline Hypertension & $38(42.2)$ & $6(75.0)$ & 0.135 \\
\hline Ischemic heart disease & $10(11.1)$ & $3(37.5)$ & 0.070 \\
\hline Cerebrovascular disease & $9(10.0)$ & $1(12.5)$ & 0.592 \\
\hline \multicolumn{4}{|l|}{ Medication, $N(\%)$} \\
\hline Aspirin & $21(23.3)$ & $2(25.0)$ & 1.000 \\
\hline Warfarin & $4(4.4)$ & $0(0.0)$ & 1.000 \\
\hline Clopidogrel & $5(5.5)$ & $3(37.5)$ & 0.017 \\
\hline ISS, mean (SD) & $18.6(8.6)$ & $21.2(4.4)$ & 0.405 \\
\hline ISS > 19, $N(\%)$ & $37(41.1)$ & $5(62.5)$ & 0.282 \\
\hline Brain AIS, mean (SD) & $3.7(1.1)$ & $4.5(0.5)$ & 0.080 \\
\hline AIS $>4, N(\%)$ & $18(20.0)$ & $4(50.0)$ & 0.073 \\
\hline SBP on admission, mean (SD), $\mathrm{mmHg}$ & $150.7(31.7)$ & $164.3(23.2)$ & 0.240 \\
\hline Heart rate on admission, mean (SD), beats/min & $85.9(16.2)$ & $96.5(24.2)$ & 0.095 \\
\hline \multicolumn{4}{|l|}{ Result of the first brain CT, $N(\%)$} \\
\hline Epidural hematoma & $16(17.7)$ & $1(12.5)$ & 1.000 \\
\hline Subdural hematoma & $51(56.6)$ & $7(87.5)$ & 0.136 \\
\hline Subarachnoid hemorrhage & $50(55.5)$ & $3(37.5)$ & 0.464 \\
\hline Hemorrhagic contusion & $46(51.1)$ & $3(37.5)$ & 0.715 \\
\hline Intraventricular hemorrhage & $6(6.6)$ & $0(0.0)$ & 1.000 \\
\hline Diffuse axonal injury & $5(5.5)$ & $0(0.0)$ & 1.000 \\
\hline Skull fracture & $30(33.3)$ & $3(37.5)$ & 1.000 \\
\hline Base of skull fracture & $5(5.5)$ & $1(12.5)$ & 0.409 \\
\hline Midline shift, mean (SD), mm & $0.67(1.5)$ & $4.25(2.1)$ & \\
\hline Midline shift, median, mm & 0 & 3.5 & 0.0015 \\
\hline Midline shift > $2 \mathrm{~mm}, N(\%)$ & $11(12.2)$ & $5(62.5)$ & 0.003 \\
\hline Duration from injury to the first brain CT, median, $\mathrm{hr}$ & 2.9 & 1.2 & 0.495 \\
\hline Duration from injury to routine repeated brain CT, median, hr & 46.9 & 38.7 & 0.603 \\
\hline
\end{tabular}

TABLE 3: Determination of odd ratio and $P$ value in neurosurgical treatment group in mild TBI.

\begin{tabular}{lccc}
\hline Variables & Odd ratio & $95 \%$ CI & $P$ value \\
\hline Subdural hematoma & 5.3 & $0.63-45.33$ & 0.136 \\
Hypertension & 4.1 & $0.78-21.46$ & 0.135 \\
AIS $>4$ & 4.0 & $0.91-17.55$ & 0.073 \\
Ischemic heart disease & 4.8 & $0.99-23.19$ & 0.070 \\
Clopidogrel & 10.2 & $1.87-55.38$ & 0.017 \\
Midline shift $>$ 2 mm & 11.9 & $2.50-57.20$ & 0.003 \\
Emergency brain CT on neurological deterioration & 30.0 & $3.46-280.83$ & $<0.001$ \\
\hline
\end{tabular}

\section{Discussion}

Brain CT is the investigation of choice to determine the severity and type of TBI cases. It is also the objective tool to evaluate the risk status for this patient group; however, to follow up duration with repeated brain CT is still controversial. Progressive hemorrhagic injury from repeated brain CT was reported about 32.3-43.6\% [1-4] in spite of no neurological change in TBI cases. There were several studies stated about controversy in clinical valuable as a 


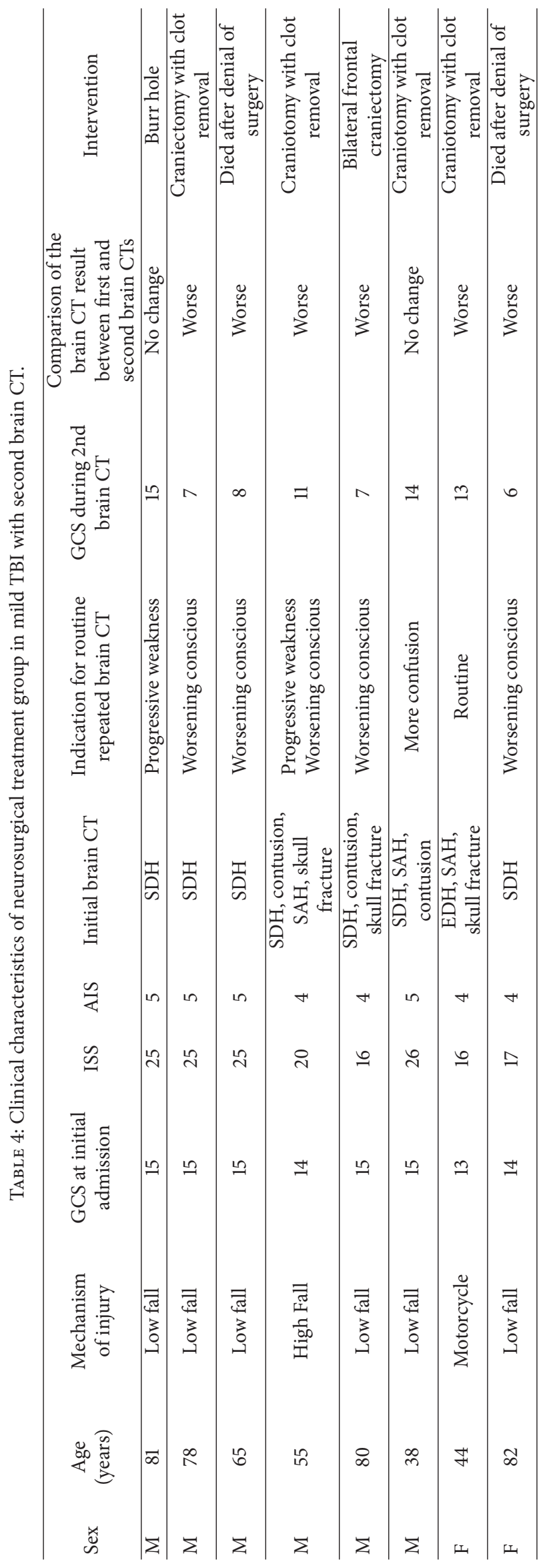


TABLE 5: Comparison of the result of routine repeated brain CT versus initial brain CT in mild TBI.

\begin{tabular}{lcccc}
\hline Traumatic brain injury & \multicolumn{2}{c}{$\begin{array}{c}\text { Result after routine repeated brain CT } \\
\text { No change }\end{array}$} & Worsened & Total \\
\hline Mild TBI & 24 & 38 & 36 & 98 \\
\hline
\end{tabular}

TABLE 6: Comparison of the result of routine repeated brain CT versus initial brain CT in moderate and severe TBI group.

\begin{tabular}{|c|c|c|c|c|}
\hline \multirow{2}{*}{ Traumatic brain injury } & \multicolumn{3}{|c|}{ Result after routine repeated brain CT } & \multirow{2}{*}{ Total } \\
\hline & Improved & No change & Worsened & \\
\hline Moderate TBI & 6 & 5 & 8 & 19 \\
\hline Severe TBI & 4 & 7 & 17 & 28 \\
\hline Total & 10 & 12 & 25 & 47 \\
\hline
\end{tabular}

routine serial brain CT without neurological examination [512]. In this present study, the result revealed that less than $10 \%$ of mild TBI underwent neurosurgical intervention. In addition, trips to CT scanner may be associated with adverse events such as extubation or cardiac arrest [13]. And also routine repeated brain CT increases patient exposure to more radiation may increase risks of cancer [14]. Some previous studies have suggested that repeated brain CT is unnecessary in patients who remain neurologically stable $[3,5-7,15,16]$. Others argue that repeated imaging is necessary because brain injuries can progress without neurologic changes [812]. However, a number of objective factors might help the physicians to make some decision for patient treatment plan or RSCM. Some interesting factors in this study including use of clopidogrel or brain CT revealing midline shift $>2 \mathrm{~mm}$ were the predicting factors for neurosurgical intervention indication. These 2 factors might be used to identify high risk patient for repeating brain CT. Regarding RSCM, the risk status is the main concern and should be an objective factor based on patient safety. Because of brain CT technology, it is an objective option to determine traumatic brain injury risk status. But, in this present study, the chance or clinical benefit to utilize repeated brain CT to determine progressive hemorrhagic injury in mild TBI patient is not compulsory to use as routine or without neurological examination as mentioned above. In mild TBI cases, brain CT should be realized that initial brain CT within 3 hours of injury is considered too early and recommended to repeated brain CT again within 12 hours $[17,18]$. Otherwise, the physician would miss and could not detect progressive brain lesion. In moderate and severe TBI cases, CT scans are usually obtained within a few hours after injury. The subsequent scans in these groups will reveal different findings and clinical deterioration also plays a great role in the decision-making. These patients usually have worse outcomes regardless of whether surgery is performed [18]. In conclusion, the clinical recommendation for an interval of repeated brain CT should be $8-12$ hours after injury [17]. In recent times, more elderly patients and antiplatelet or anticoagulation patients are the major concern. And also there is higher prevalence of chronic and multiple illnesses and too early brain CT investigation so one should be cautious of the missing or delayed progressive intracranial hematoma such as chronic subdural hemorrhage in aging patients. Because of this, repeated brain CT should be considered as a lesson learned and be your own custom case.

\section{Conclusion}

Routine repeated brain CT for RSCM had no clinical benefit in patient with mild traumatic brain injury without neurological examination. Clopidogrel and midline shift more than $2 \mathrm{~mm}$ are the clinical predicted factors to indicate neurosurgical intervention after repeated brain CT.

\section{Additional Points}

Strengths and Limitations of This Study

(i) This present study is the observational single-center, retrospective design so the recruited cases and clinical management are the confounding variables.

(ii) Being a retrospective study, this may confine only in our clinical findings and management. Consequently, our discussion is not the clinical guideline or consensus for overall management of TBI patients.

(iii) Some missing data most likely to occur in this present study and also the confounders might affect the difference of the factors.

(iv) Regarding the definitions of neurological examination, we did not differentiate between different degrees of weakness or deficit.

(v) The risk-stratified care management is the process of management for a risk status evaluation to help patients achieve the best quality of care and to prevent acceleration to higher-risk categories and higher associated costs.

\section{Competing Interests}

The authors declared no competing interests.

\section{Authors' Contributions}

Sorayouth Chumnanvej contributed to the concept and design of the article, analysis and interpretation of data, 
drafting of the article, and revising and final approval of the version to be published. Preeda Sumritpradit and Thitipong Setthalikhit contributed to analysis and interpretation of data and drafting of the article.

\section{References}

[1] M. Oertel, D. F. Kelly, D. McArthur et al., "Progressive hemorrhage after head trauma: predictors and consequences of the evolving injury," Journal of Neurosurgery, vol. 96, no. 1, pp. 109116, 2002.

[2] A. Chao, J. Pearl, P. Perdue et al., "Utility of routine serial computed tomography for blunt intracranial injury," The Journal of Trauma, vol. 51, no. 5, pp. 870-876, 2001.

[3] Z. C. Sifri, A. T. Homnick, A. Vaynman et al., "A prospective evaluation of the value of repeat cranial computed tomography in patients with minimal head injury and an intracranial bleed," Journal of Trauma-Injury, Infection and Critical Care, vol. 61, no. 4, pp. 862-867, 2006.

[4] Y. B. Roka, P. Kumar, P. Bista et al., "Role of repeat CT scan head in initially inoperable cases of traumatic head injury," Nepal Medical College Journal, vol. 10, no. 4, pp. 225-229, 2008.

[5] G. C. Velmahos, A. Gervasini, L. Petrovick et al., "Routine repeat head CT for minimal head injury is unnecessary," Journal of Trauma, vol. 60, no. 3, pp. 494-501, 2006.

[6] C. W. Washington and R. L. Grubb Jr., "Are routine repeat imaging and intensive care unit admission necessary in mild traumatic brain injury?" Neurosurgery, vol. 116, pp. 549-557, 2012.

[7] K. R. Abdelfattah, A. L. Eastman, K. N. Aldy et al., "A prospective evaluation of the use of routine repeat cranial CT scans in patients with intracranial hemorrhage and GCS score of 13 to 15," Journal of Trauma and Acute Care Surgery, vol. 73, no. 3, pp. 685-688, 2012.

[8] T. K. Bee, L. J. Magnotti, M. A. Croce et al., "Necessity of repeat head CT and ICU monitoring in patients with minimal brain injury," The Journal of Trauma, vol. 66, no. 4, pp. 1015-1018, 2009.

[9] H.-K. Park, W.-I. Joo, C.-K. Chough, C. L.-B. Cho, K.-J. Lee, and H.-K. Rha, "The clinical efficacy of repeat brain computed tomography in patients with traumatic intracranial haemorrhage within 24 hours after blunt head injury," British Journal of Neurosurgery, vol. 23, no. 6, pp. 617-621, 2009.

[10] S. C. Stein, A. Fabbri, and F. Servadei, "Routine serial computed tomographic scans in mild traumatic brain injury: when are they cost-effective?" The Journal of Trauma, vol. 65, no. 1, pp. 66-72, 2008.

[11] B. W. Thomas, V. A. Mejia, R. A. Maxwell et al., "Scheduled repeat CT scanning for traumatic brain injury remains important in assessing head injury progression," Journal of the American College of Surgeons, vol. 210, no. 5, pp. 824-830, 2010.

[12] M. C. Wang, K. F. Linnau, D. L. Tirschwell, and W. Hollingworth, "Utility of repeat head computed tomography after blunt head trauma: a systematic review," Journal of Trauma-Injury, Infection and Critical Care, vol. 61, no. 1, pp. 226-233, 2006.

[13] H. E. Stearley, "Patients' outcomes: intrahospital transportation and monitoring of critically ill patients by a specially trained ICU nursing staff," American Journal of Critical Care, vol. 7, no. 4, pp. 282-287, 1998.

[14] E. Ron, "Cancer risks from medical radiation," Health Physics, vol. 85, no. 1, pp. 47-59, 2003.
[15] F. F. Connon, B. Namdarian, J. L. C. Ee, K. J. Drummond, and J. A. Miller, "Do routinely repeated computed tomography scans in traumatic brain injury influence management? A prospective observational study in a level 1 trauma center," Annals of Surgery, vol. 254, no. 6, pp. 1028-1031, 2011.

[16] Z. C. Sifri, N. Nyak, A. T. Homnick, A. A. Mohr, P. Yonclas, and D. H. Livingston, "Utility of repeat head computed tomography in patients with an abnormal neurologic examination after minimal head injury," Journal of Trauma-Injury, Infection and Critical Care, vol. 71, no. 6, pp. 1605-1610, 2011.

[17] C. V. R. Brown, G. Zada, A. Salim et al., "Indications for routine repeat head computed tomography (CT) stratified by severity of traumatic brain injury," Journal of Trauma-Injury, Infection and Critical Care, vol. 62, no. 6, pp. 1339-1344, 2007.

[18] F. Servadei, G. D. Murray, K. Penny et al., "The value of the 'worst' computed tomographic scan in clinical studies of moderate and severe head injury," Neurosurgery, vol. 46, no. 1, pp. 70-77, 2000. 


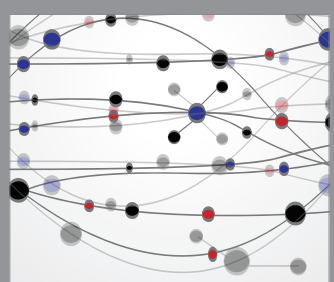

The Scientific World Journal
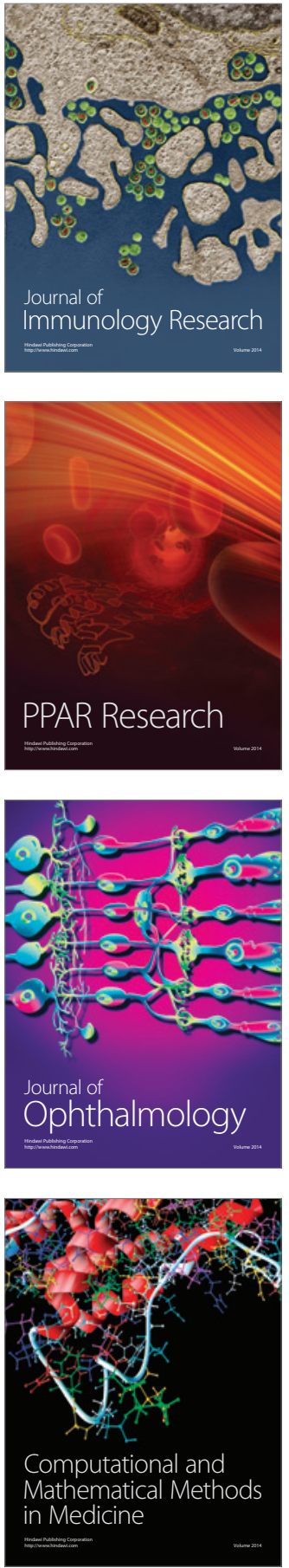

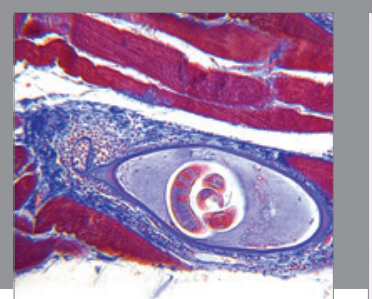

Gastroenterology Research and Practice

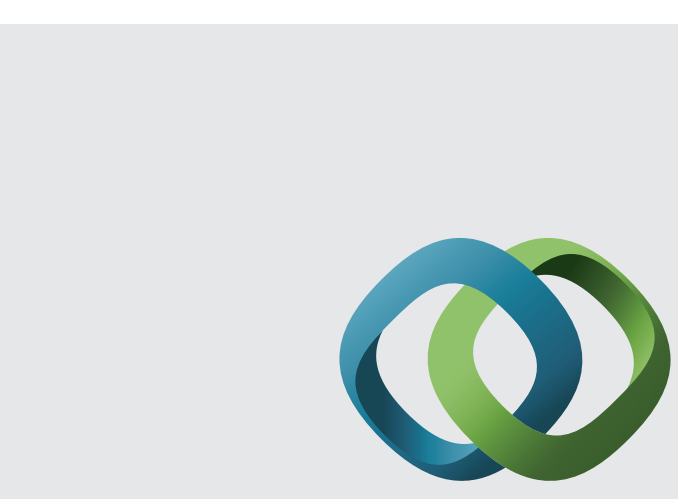

\section{Hindawi}

Submit your manuscripts at

http://www.hindawi.com
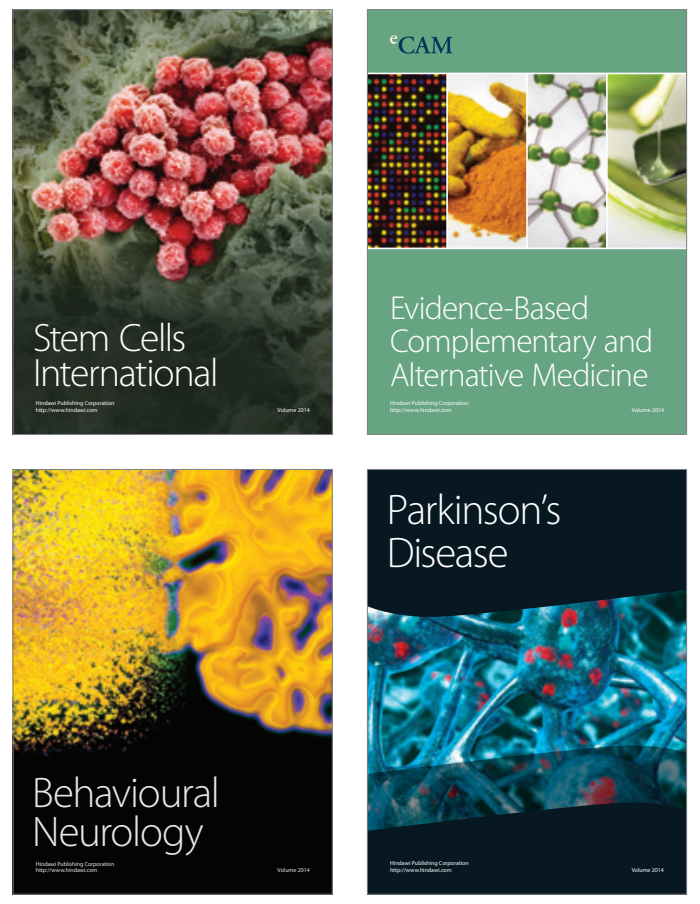
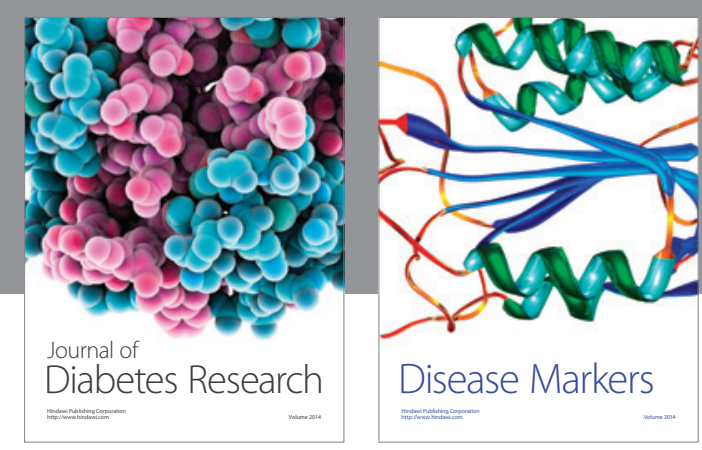

Disease Markers
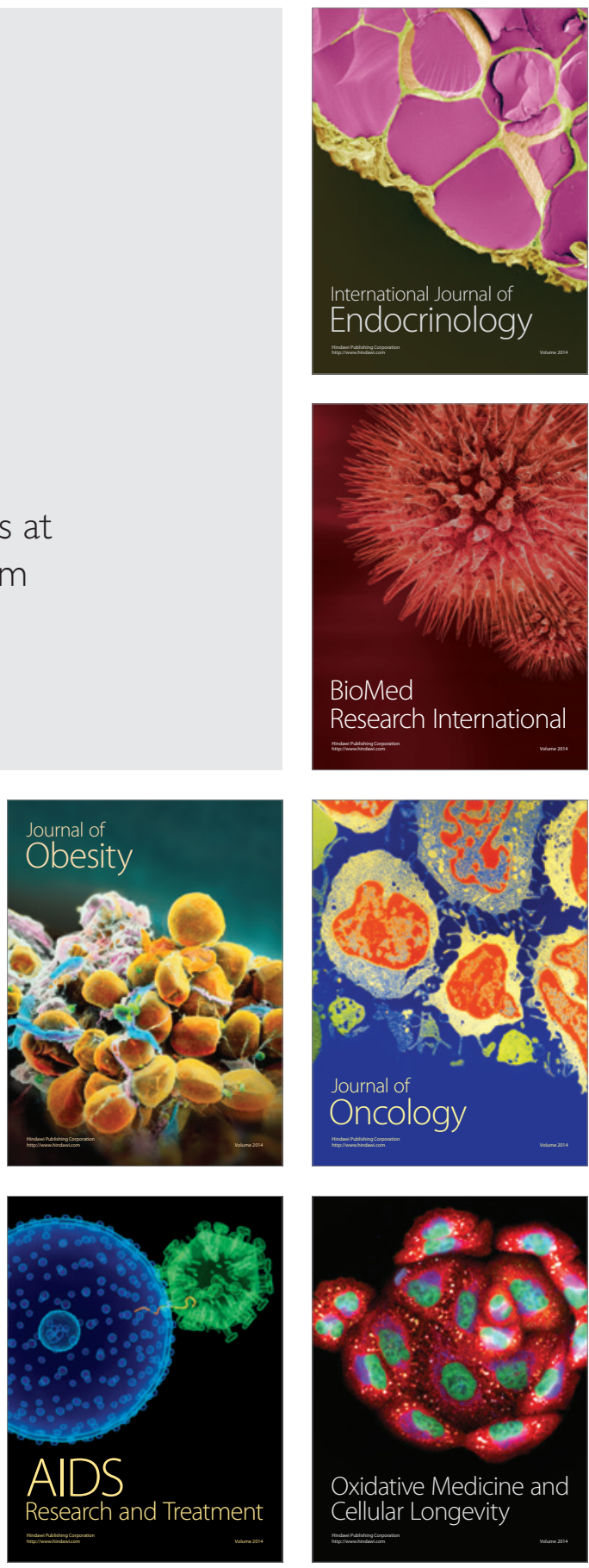